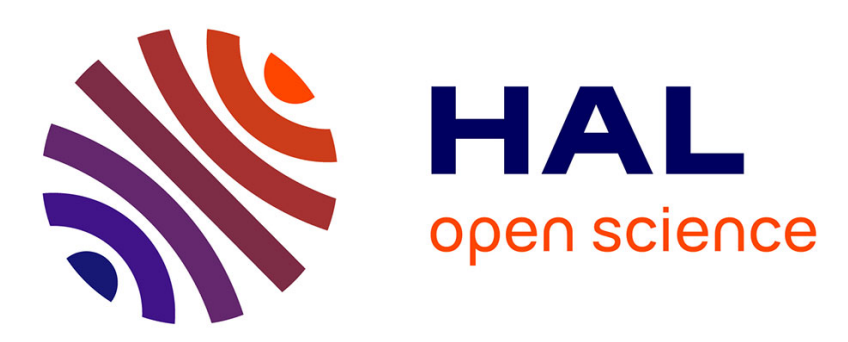

\title{
Interconnected Observers for a Powered Two-Wheeled Vehicles: Both Lateral and Longitudinal Dynamics Estimation
}

\author{
Majda Fouka, Lamri Nehaoua, Hichem Arioui, Saïd Mammar
}

\section{To cite this version:}

Majda Fouka, Lamri Nehaoua, Hichem Arioui, Saïd Mammar. Interconnected Observers for a Powered Two-Wheeled Vehicles: Both Lateral and Longitudinal Dynamics Estimation. International Conference on Networking, Sensing and Control (ICNSC 2019), May 2019, Banff, Canada. pp.163-168, 10.1109/ICNSC.2019.8743290 . hal-02071696

\section{HAL Id: hal-02071696 https://hal.science/hal-02071696}

Submitted on 18 Mar 2019

HAL is a multi-disciplinary open access archive for the deposit and dissemination of scientific research documents, whether they are published or not. The documents may come from teaching and research institutions in France or abroad, or from public or private research centers.
L'archive ouverte pluridisciplinaire HAL, est destinée au dépôt et à la diffusion de documents scientifiques de niveau recherche, publiés ou non, émanant des établissements d'enseignement et de recherche français ou étrangers, des laboratoires publics ou privés. 


\title{
Interconnected Observers for a Powered Two-Wheeled Vehicles: Both Lateral and Longitudinal Dynamics Estimation
}

\author{
M. Fouka, L. Nehaoua, H. Arioui and S. Mammar \\ IBISC Laboratory. Evry Val d'Essonne University, Evry 91000, France. \\ Email: \{majda.fouka, lamri.nehaoua, hichem.arioui, said.mammar\}@ univ-evry.fr.
}

\begin{abstract}
The paper focuses on the accurate estimation of the powered two wheelers vehicle states, including both the longitudinal and lateral dynamics. The examination of road crashes statistic reveals that loses of control is responsible for the most motorcycle accidents. Motivated by the need of observers to acquire certain states used in safety and control systems to prevent possible dangerous situation, this work investigates the design of an interconnected observers. First, the linear parameter varying (LPV) of the two-sub models of the PTWv motion are transformed into a Takagi-Sugeno (TS) form. Secondly, the observer convergence study is based on Lyapunov theory associated with the Input to State Practical Stability (ISpS) to guaranty boundedness of the state estimation errors. Further, sufficient conditions are given in terms of linear matrix inequalities (LMIs). Finally, observer performances are tested and compared to the motorcycle model states and several simulation cases are provided to highlight the effectiveness of the suggested method using motorcycle Simulator Software BikeSim ${ }^{\odot}$.
\end{abstract}

Index Terms-Interconnected Observer, Motorcycle Safety, longitudinal and lateral dynamics.

\section{INTRODUCTION}

The integration of Advanced Rider Assistance Systems (ARAS) and Intelligent Transportation Systems (ITS) for powered two wheeled vehicles (PTWv) is one of the forward objective in automakers and suppliers to help make partially automated riding and serve as safety system to support/alert the rider of potentially hazardous situations [1], [2]. In the last decade, electronic driving aids are becoming more and more a research focus to ensure optimal riding behaviour [3], [4]. A thorough improvement of this systems requires accurate motorcycle states informations. However, the measurement by sensors of all dynamic states and inputs with conventional sensors is inconceivable for economic or technical reasons (the price or the feasibility of some sensors). Indeed, virtual sensor is one of the key research fields using model-based estimators to overcome previous shortcomings in order to provide estimates of unmeasured states and relevant parameters of the PTWv's dynamics. Several methods were proposed to estimate motorcycle dynamics states, extensive research efforts have been given to the development of state observer for lateral dynamics to enhance handling and stability of PTWv, one can cite [5]-[8].

978-1-7281-0084- 5/19/31.002019 IEEE
The study of rectilinear motion highlights certain dynamic aspects that are also important for safety, such as the motorcycle's behavior during braking with possible forward overturning, and in acceleration, with possible wheeling [9]. Hard acceleration or braking is an unsafe riding, is often a cause of craches for motorcycles. In this context, estimate the longitudinal motion for PTWv is essential to develop braking and traction active safety and control systems. Several research have been proposed to wheel slip control [10], [11]. However, vary few works deal with states estimation in-plane dynamic states [12], [13], based on the estimation of the lateral dynamics, the author used an algebraic reformulation of the unknown variables and numerical differentiators to reconstruct the longitudinal tire-road forces. In almost references, the estimation of the PTWv dynamics is done by considering restrictive assumptions with respect to riding practice: decoupling motion or independent behaviour and/or a constant longitudinal speed. The coupling motion of the lateral and longitudinal dynamics of two wheeled vehicle have not received much attention in the literature.

This paper presents a method for synthesizing an interconnected LPV observer for simultaneous estimation of the lateral and longitudinal dynamics of the two wheelers. This method is based on the decomposition of motorcycle model into two LPV subsystems, then each LPV subsystems model of the vehicle is transformed into Takagi-Sugeno (TS), the result is formalized using Lyapunov theory and the Input to State Practical Stability (ISpS) formulated as an optimization problem under Linear Matrix Inequalities (LMI) aiming to minimize the error estimation bound.

\section{TWO-WHEELED DYNAMICS DESCRIPTION}

The performance study of PTWv focuses on: the longitudinal dynamics which refers to the vehicle's ability to accelerate, brake and to develop traction in order to overcome obstacles. Therefore, the lateral dynamic study quantifies the vehicle's ability to support lateral accelerations, it can be characterized also by its ability to develop lateral forces to follow a steering rider input. In most situation, the motorcycle develop a combined scenarios and applied simultaneously longitudinal forces (braking or acceleration) and lateral forces. 


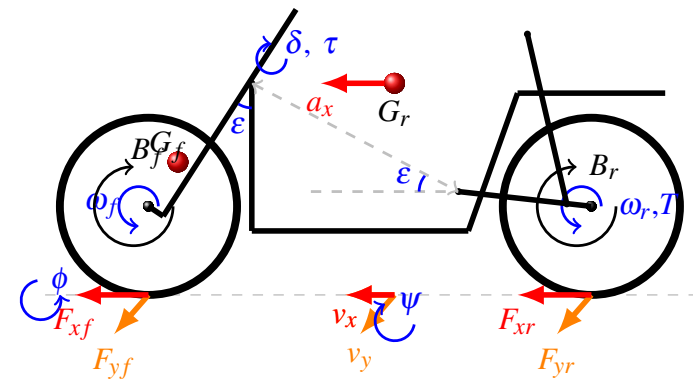

Fig. 1: Kinematic representation of PTWv.

\section{A. Longitudinal dynamics of PTWv}

The model of the longitudinal dynamics under the influence of a coupled motions, describing the tires and braking systems are given by the following equations :

$$
\left\{\begin{aligned}
M\left(v_{x}-v_{y} \dot{\psi}\right) & =F_{x f}+F_{x r}-C_{d} v_{x}^{2}-F_{r r} \\
i_{f y} \dot{\omega}_{f} & =-R_{f} F_{x f}+B_{f} \\
i_{r y} \dot{\omega}_{r} & =-R_{r} F_{x r}+T+B_{r}
\end{aligned}\right.
$$

- $F_{a}=C_{d} v_{x}^{2}$ is the aerodynamic force, $C_{d}$ : the drag coefficient.

- $F_{r r}=f_{\omega} F z$ is the rolling resistance force with $f_{\omega}$ is the rolling resistance coefficient [4], $p_{r}$ is the tire pressure:

$$
f_{\omega}=\left\{\begin{array}{rll}
0.0085+\frac{0.018}{p_{r}}+\frac{1.59 \cdot 10^{-6} \cdot v_{x}^{2}}{p_{r}} & \text { if } & v_{x} \prec 165 \mathrm{~km} / \mathrm{h} \\
\frac{0.018}{p_{r}}+\frac{2.91 .10^{-6} \cdot v_{x}^{2}}{p_{r}} & \text { if } & v_{x} \succ 165 \mathrm{~km} / \mathrm{h}
\end{array}\right.
$$

- $B_{f}$ and $B_{r}$ are the braking torques applied to the front and rear tires, $T$ is the engine torque applied on rear wheel.

- $\omega_{f, r}$ is the wheel rotational speed, $i_{(f, r) y}$ the moment of inertia of the wheels, $R_{f, r}$ are the wheel radius.

The longitudinal forces dynamics are given by:

$$
\frac{\sigma_{i}}{v_{x}} \dot{F}_{x i}=-F_{x i}+C_{i} \lambda_{i}, \quad i=(f, r)
$$

where, $\lambda_{i}=\left(R_{i} \omega_{i}-v_{x}\right) \cdot \rho_{i}$ and $\rho_{i}=\frac{1}{\max \left(R_{i} \omega_{i}, v_{x}\right)} . \lambda_{i}$ are the longitudinal slip angles, $C_{i}$ are the tire longitudinal stiffness, $\sigma_{i}$ are tire relaxation lengths.

\section{B. Lateral dynamics of PTWv}

The lateral dynamics of the PTWv is represented as a set of two bodies allowing the simulation of 4 DoF: yaw, roll, steering and lateral motion figure 1 . The lateral dynamics can be expressed by the following equations [14]:

$$
\left\{\begin{aligned}
\sum F_{y} & =e_{33} \dot{v}_{y}+e_{34} \ddot{\psi}+e_{35} \ddot{\phi}+e_{36} \ddot{\delta}-a_{34} \dot{\psi} \\
\sum M_{z} & =e_{34} \dot{\nu}_{y}+e_{44} \ddot{\psi}+e_{45} \ddot{\phi}+e_{46} \ddot{\delta}-a_{44} \dot{\psi}-a_{45} \dot{\phi}-a_{46} \dot{\delta} \\
\sum M_{x} & =e_{35} \dot{\nu}_{y}+e_{45} \ddot{\psi}+e_{55} \ddot{\phi}+e_{56} \ddot{\delta}-a_{54} \dot{\psi}-a_{55} \dot{\delta} \\
\sum M_{s} & =e_{36} \dot{y_{y}}+e_{46} \ddot{\psi}+e_{56} \ddot{\phi}+e_{66} \ddot{\tilde{\delta}}-a_{64} \dot{\psi}-a_{65} \dot{\phi}-a_{66} \dot{\delta} \\
\dot{F}_{y f} & =a_{71} \phi+a_{72} \delta+a_{73} v_{y}+a_{74} \dot{\psi}+a_{76} \dot{\delta}+a_{77} F_{y f} \\
F_{y r} & =a_{81} \phi+a_{83} v_{y}+a_{84} \dot{\psi}+a_{88} F_{y r}
\end{aligned}\right.
$$

where:

$$
\left\{\begin{array}{l}
\sum F_{y}=F_{y f}+F_{y r}=M a_{y} \\
\sum M_{z}=a_{47} F_{y f}+a_{48} F_{y r} \\
\sum M_{x}=a_{51} \sin (\phi)+a_{52} \sin (\delta) \\
\sum M_{s}=a_{61} \sin (\phi)+a_{62} \sin (\delta)+a_{67} F_{y f}+\tau
\end{array}\right.
$$

Where $(\phi, \delta, \psi, \dot{\phi}, \dot{\delta}, \dot{\psi})$ denote the roll, steering, yaw angles and respectively their time derivatives, whereas $v_{y}$ is the lateral velocity, $F_{y f}$ and $F_{y r}$ are the the cornering front and rear forces respectively, and $\tau$ is the rider torque applied to the handle bar. The pneumatic forces $F_{y f}$ and $F_{y r}$, are generated when there is simultaneously side slip angles $\alpha_{f}$ and $\alpha_{r}$ and camber angles $\gamma_{f}$ and $\gamma_{r}$, expressed by:

$$
\frac{\sigma_{i}}{v_{x}} \dot{F}_{y i}=-F_{y i}-C_{i 1} \alpha_{i}+C_{i 2} \gamma_{i}, \quad i=(f, r)
$$

where $\alpha_{f}=\left(\frac{v_{y}+l_{f} \dot{\psi}-\eta \dot{\delta}}{v_{x}}\right)-\delta \cos (\varepsilon), \alpha_{r}=\left(\frac{v_{y}-l_{r} \dot{\psi}}{v_{x}}\right), \gamma_{f}=$ $\phi+\delta \sin (\varepsilon)$ and $\gamma_{r}=\phi . C_{i 1}$ and $C_{i 2}$ refer to the tire forces coefficients (stiffness and camber coefficients), $\varepsilon$ refers to the caster angle, $\eta$ is the mechanical trail, $l_{f}$ (resp. $l_{r}$ ) is distance between the center of mass and the front and rear axis.

\section{INTERCONNECTED MOTORCYCLE MODEL \& COUPLING ANALYSIS}

The models established in the previous section allow to take into account the most important variable which are obviously necessary in order to simulate the correct riding behavior.

\section{A. State Space Representation}

In this work, the first sub-system of the rectilinear motion describing the rotations of the tires will be consider varying. From equations 1 and 3 , one have a LPV model with $\zeta_{1}(t)$ refers to $\left[v_{x}, \omega_{f}, \omega_{f}, F_{x f}, F_{x r}\right]^{T}$ and $u_{B}=\left[B_{f}, B_{r}+T\right]^{T}$. Thus, the motorcycle longitudinal dynamics will be modelled by the following sub-state space model:

$$
\dot{\zeta}_{1}(t)=\bar{A}\left(\zeta_{1}\right) \zeta_{1}(t)+\bar{B} u_{B}(t)+\bar{D}\left(\zeta_{2}\right) \zeta_{2}(t)
$$

In this model, longitudinal velocity $v_{x}$, longitudinal front and rear stiffness $\rho_{f, r}$ may be seen as external varying parameters. The state model of the lateral motion is also a LPV model. From equations (4), one have a model with $\zeta_{2}(t)=$ $\left[\phi, \delta, v_{y}, \dot{\psi}, \dot{\phi}, \dot{\delta}, F_{y f}, F_{y r}\right]^{T}$ and $u_{\tau}=\tau(t)$, the motorcycle lateral dynamics will be modelled by the following system:

$$
\dot{\zeta}_{2}(t)=\breve{A}\left(\zeta_{1}\right) \zeta_{2}(t)+\breve{B} u_{\tau}(t)+\breve{D} \zeta_{1}(t)
$$

For the PTWv, the output vector is $y_{x}=\left[\omega_{f}, \omega_{r}, a_{x}\right], y_{y}=$ $\left[\delta, \dot{\psi}, \dot{\phi}, a_{y}\right]$. Thus, the motorcycle interconnected dynamics will be modelled by the following state space model:

$$
\left\{\begin{array}{c}
\dot{\zeta}_{1}(t)=\bar{A}\left(\zeta_{1}\right) \zeta_{1}(t)+\bar{B} u_{B}(t)+\bar{D} \zeta_{2}(t) \\
\dot{\zeta} 2(t)=\breve{A}\left(\zeta_{1}\right) \zeta_{2}(t)+\breve{B} u_{\tau}(t)+\breve{D} \zeta_{1}(t) \\
y_{x}(t)=\bar{C} \zeta_{1}(t) \\
y_{y}(t)=\breve{C} \zeta_{2}(t)
\end{array}\right.
$$

where : $\xi(t)=\left[\begin{array}{lll}\zeta_{1}(t) & \zeta_{2}(t)\end{array}\right]^{T}$ and $C=\left[\begin{array}{ll}\bar{C} & \breve{C}\end{array}\right]^{T}$. where $\zeta_{1}(t) \in \mathbb{R}^{5}$ and $\zeta_{2}(t) \in \mathbb{R}^{8}$ are the state vector, and $y_{x}(t) \in \mathbb{R}^{3}$ and $y_{y}(t) \in \mathbb{R}^{5}$ the output vector; $\bar{F}(\cdot)$ and $\breve{F}(\cdot)$ are nonlinear functions. 


\section{B. Exact T-S model of a motorcycle nonlinear model}

From the well-known sector nonlinearity approach [15], the LPV interconnected model can be written in an exact TS representation with 8 sub-models for the longitudinal model and 2 sub-model for lateral model. Indeed, the scheduling variables in TS systems, are:

1) Longitudinal model, the number 8 of sub-models comes from the fact that 3 nonlinearities, the membership functions of the fuzzy sets are defined as

$$
\begin{aligned}
& z_{1}=v_{x}, \quad z_{2}=\rho_{f}, \quad z_{3}=\rho_{r} \\
& z_{1}^{\min } \leq z_{1} \leq z_{1}^{\max } \quad z_{2}^{\min } \leq z_{2} \leq z_{2}^{\max } \quad z_{3}^{\min } \leq z_{3} \leq z_{3}^{\max }
\end{aligned}
$$

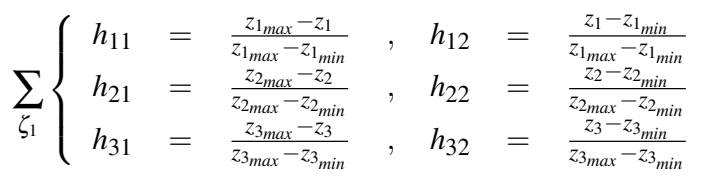

The variables $\mu_{i}(\rho)$ are computed as follows:

$$
\left\{\begin{array}{l}
\mu_{1}=h_{11} \cdot h_{21} \cdot h_{31}, \quad \mu_{2}=h_{12} \cdot h_{21} \cdot h_{31} \\
\mu_{3}=h_{11} \cdot h_{22} \cdot h_{31}, \mu_{4}=h_{12} \cdot h_{22} \cdot h_{31} \\
\mu_{5}=h_{11} \cdot h_{21} \cdot h_{32}, \mu_{6}=h_{12} \cdot h_{21} \cdot h_{32} \\
\mu_{7}=h_{11} \cdot h_{22} \cdot h_{32}, \mu_{8}=h_{12} \cdot h_{22} \cdot h_{32}
\end{array}\right.
$$

2) Lateral model: 2 of sub-models comes from 1 nonlinearity, the motorcycle, is stable only for a range of longitudinal velocities $v_{x}$, the bounds of the premise variables are given by:

$$
\sum_{\zeta_{2}}\left\{\begin{array}{l}
\vartheta_{1}=\frac{v_{x_{\max }}-v_{x}}{v_{x_{\max }}-v_{x_{\min }}} \\
\vartheta_{2}=\frac{v_{x}-v_{x_{\min }}}{v_{x_{\max }}-v_{x_{\min }}}
\end{array}\right.
$$

The variables $\mu_{i}$ and $\vartheta_{j}$ are called the weighing functions and they must satisfy the following convex sum property:

$$
\left\{\begin{array}{cc}
0 \leq \mu_{i}\left(z_{1}, z_{2}, z_{3}\right) & \leq 1 \\
0 \leq \vartheta_{j}\left(v_{x}\right) & \leq 1 \\
\sum_{i=1}^{8} \mu_{i}\left(z_{1}, z_{2}, z_{3}\right) & =1 \\
\sum_{j=1}^{2} \vartheta_{j}\left(v_{x}\right) & =1
\end{array}\right.
$$

Applying the sector nonlinearity, the system (6) is rewritten:

$$
\left\{\begin{array}{c}
\left.\dot{\zeta}_{1}(t)=\sum_{i=1}^{p_{1}} \mu_{i}\left(\zeta_{1}(t)\right)\left(\bar{A}_{i} \zeta_{1}(t)+\bar{D}_{i} \zeta_{2}(t)+\bar{B}_{i} u_{T}(t)\right)\right) \\
y_{x}(t)=\bar{C} \zeta_{1}(t), \quad p_{1}=8 \\
\dot{\zeta}_{2}(t)=\sum_{j=1}^{p_{2}} \vartheta_{j}\left(\zeta_{1}(t)\right)\left(\breve{A}_{j} \zeta_{2}(t)+\breve{D}_{j} \zeta_{1}(t)+\breve{B}_{j} u_{\tau}(t)\right) \\
y_{y}(t)=\bar{C} \zeta_{2}(t), \quad p_{2}=2
\end{array}\right.
$$

\section{OBSERVER DESIGN}

\section{A. Preliminary}

Assumption 1: Throughout the paper, the following nonrestrictive assumptions are considered:

- Assume that, for the design of each observer, the states of other subsystems are available.

- The state vector $\left(\zeta_{1}\right)$ and $\left(\zeta_{2}\right)$ of the two models are considered bounded,

- The pair $\left(\breve{A}\left(\zeta_{1}\right), \breve{C}\right)$ and $\left(\bar{A}\left(\zeta_{2}\right), \bar{C}\right)$ are observable.

Lemma 1: [16] Consider $\Upsilon$ and $\Xi$ matrices with appropriate dimensions. For every matrix $\Lambda>0$, the property holds:

$$
\Upsilon^{T} \Xi+\Xi^{T} \Upsilon \leq \Upsilon^{T} \Lambda \Upsilon+\Xi^{T} \Lambda^{-1} \Xi
$$

Lemma 2 (The Schur's lemma): [16] Given the following matrices $\Upsilon, \Xi$ and $\boldsymbol{\kappa}$, with appropriate dimensions, where $\Upsilon=$ $\Upsilon^{T}$ and $\boldsymbol{\aleph}=\boldsymbol{\aleph}^{T}$, thus:

$$
\left[\begin{array}{cc}
\Upsilon & \Xi \\
\Xi^{T} & \boldsymbol{\aleph}
\end{array}\right]<0 \Leftrightarrow\left\{\begin{array}{c}
\boldsymbol{\aleph}<0 \\
\Upsilon-\Xi \boldsymbol{\aleph}^{-1} \Xi^{T}<0
\end{array}\right.
$$

Definition 1: [17] The state estimation error dynamics verifies the Input To State Practical Stability (ISpS) if there exists a $\mathscr{K} \mathscr{L}$ function $\beta: \mathbb{R}^{n} \times \mathbb{R} \longrightarrow \mathbb{R}$, a $\mathscr{K}$ function $\alpha: \mathbb{R} \longrightarrow \mathbb{R}$ such that for each input $\Delta(t)$ satisfying $\|\Delta(t)\|_{\infty}<\infty$ and each initial conditions $e(0)$, the trajectory of the errors associated to $e(0)$ and $\Delta(t)$ satisfies

$$
\|e(t)\|_{2} \leq \beta(\|e(0)\|, t)+\alpha\left(\|\Delta(t)\|_{\infty}\right)
$$

An overall scheme of the system/observer structure is given in figure 2 .

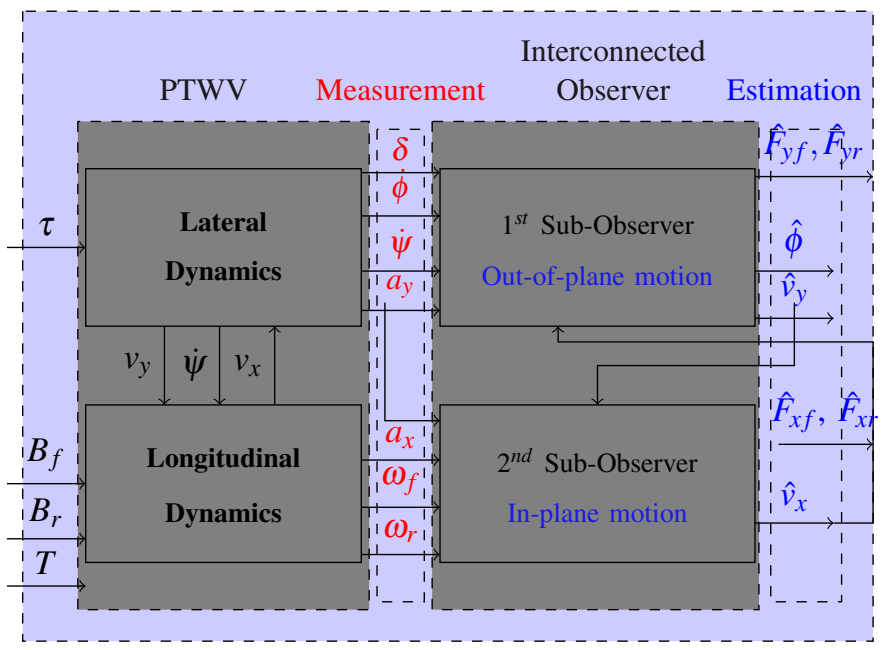

Fig. 2: General diagram of the interconnected estimation.

\section{B. State estimation}

The following observer is proposed. This observer is based on the interconnection between the two subsystems (14):

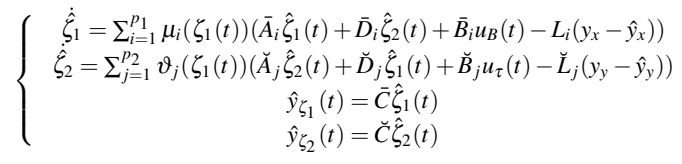

Using equations (14) and (18), the state estimation error obeys the following differential equation:

$$
\left\{\begin{array}{l}
\dot{e}_{\zeta_{1}}=\sum_{i=1}^{p_{1}} \mu_{i}\left(\zeta_{1}(t)\right)\left(\bar{\Phi}_{i} e_{\zeta_{1}}+\bar{D}_{i} e_{\zeta_{2}}\right)+\Delta_{\zeta_{1}}(t) \\
\dot{e}_{\zeta_{2}}=\sum_{j=1}^{p+2} \vartheta_{j}\left(\zeta_{1}(t)\right)\left(\bar{\Phi}_{j} e_{\zeta_{2}}+\bar{D}_{j} e_{\zeta_{1}}\right)+\Delta_{\zeta_{2}}(t)
\end{array}\right.
$$

where: $\bar{\Phi}_{i}=\left(\bar{A}_{i}-L_{i} \bar{C}\right), \breve{\Phi}_{j}=\left(\breve{A}_{j}-L_{j} \breve{C}\right), \Delta_{\zeta_{i}}(t)=\sum_{i=1}^{r_{i}}\left(\mu_{i}\left(\zeta_{1}\right)-\mu_{i}\left(\hat{\zeta}_{1}\right)\right) \bar{A}_{i} \zeta_{1}(t)$ and $\Delta_{\zeta_{2}}(t)=\sum_{i=1}^{r_{i}}\left(\vartheta_{j}\left(\zeta_{1}\right)-\vartheta_{j}\left(\hat{\zeta}_{2}\right)\right) \check{A}_{j} \zeta_{2}(t)$.

Notice that if the state estimation errors converge to zero, the terms $\Delta_{\zeta_{1}}(t)$ and $\Delta_{\zeta_{2}}(t)$ converge also towards zero. In addition, since the weighting functions are bounded and the state vector $\zeta_{1}(t)$ and $\zeta_{2}(t)$ are also bounded (see assumption $1)$, the term $\Delta_{\zeta_{i}}(t)$ are thus bounded.

By considering $e_{\zeta}=\left(e_{\zeta_{1}}, e_{\zeta_{2}}\right)=\left(\zeta_{1}-\hat{\zeta}_{1}, \zeta_{2}-\hat{\zeta}_{2}\right)$ the observers errors dynamics are given by:

$$
\dot{e}_{\zeta}=\left[\begin{array}{cc}
\sum_{j=1}^{p_{1}} \mu_{i}(\zeta) \bar{\Phi}_{i} & \mu_{i}(\zeta) \bar{D}_{i} \\
\sum_{j=1}^{p_{1}} \vartheta_{j}(\zeta) \breve{D}_{j} & \sum_{j=1}^{p_{i}} \vartheta_{j}(\zeta) \breve{\Phi}_{j}
\end{array}\right] \times e_{\zeta}+\left[\begin{array}{c}
\Delta_{\zeta_{1}}(t) \\
\Delta_{\zeta_{2}}(t)
\end{array}\right]
$$


1) Convergence analysis: To study the convergence of the the interconnected observer, the Lyapunov function is used:

$$
V(e(t))=e_{\zeta_{1}}(t)^{T} P e_{\zeta_{1}}(t)+e_{\zeta_{2}}(t)^{T} Q e_{\zeta_{2}}(t)
$$

The time-derivative of the Lyapunov function (21) is:

$$
\begin{gathered}
\dot{V}(e)=\sum_{i=1}^{p_{1}} \mu_{i}\left(\zeta_{1}\right)\left(\left(\bar{\Phi}_{i} e_{\zeta_{1}}+\bar{D}_{i} e_{\zeta_{2}}\right)^{T} P e_{\zeta_{1}}+e_{\zeta_{1}}^{T} P\left(\bar{\Phi}_{i} e_{\zeta_{1}}+\bar{D}_{i} e_{\zeta_{2}}\right)\right)+ \\
\sum_{j=1}^{p_{2}} \vartheta_{j}\left(\zeta_{1}\right)\left(\left(\breve{\Phi}_{j} e_{\zeta_{2}}+\breve{D}_{j} e_{\zeta_{\zeta_{2}}}\right)^{T} Q e_{\zeta_{2}}+e_{\zeta_{2}}^{T} Q\left(\bar{\Phi}_{j} e_{\zeta_{2}}+\breve{D}_{j} e_{\zeta_{1}}\right)\right)+ \\
e_{\zeta_{1}}^{T} P \Delta_{\zeta_{2}}+\Delta_{\zeta_{1}}^{T} P e_{\zeta_{1}}+e_{\zeta_{2}}^{T} Q \Delta_{\zeta_{2}}+\Delta_{\zeta_{2}}^{T} Q e_{\zeta_{2}}
\end{gathered}
$$

Considering $\Gamma_{i}=\bar{\Phi}_{i}^{T} P+P \bar{\Phi}_{i}$ and $\breve{\Gamma}_{j}=\breve{\Phi}_{j}^{T} Q+Q \breve{\Phi}_{j}$, we have:

$$
\begin{aligned}
& \dot{V}(e(t))= \\
& \sum_{i=1}^{p_{1}} \mu_{i}\left(\zeta_{1}\right)\left(e_{\zeta_{1}}^{T} \Gamma_{i} e_{\zeta_{1}}+e_{\zeta_{1}}^{T} P \bar{D}_{i} e_{\zeta_{2}}+e_{\zeta_{\zeta_{1}}} \bar{D}_{i}^{T} P e_{\zeta_{1}}+2 e_{\zeta_{1}}^{T} P \Delta_{\zeta_{1}}\right)+ \\
& \sum_{j=1}^{p_{2}} \vartheta_{j}\left(\zeta_{1}\right)\left(e_{\zeta_{2}}^{T} \breve{\Gamma}_{j} e_{\zeta_{1}}+e_{\zeta_{2}}^{T} Q \breve{D}_{j} e_{\zeta_{1}}+e_{\zeta_{1}}^{T} \breve{D}_{j}^{T} P e_{\zeta_{2}}+2 e_{\zeta_{2}}^{T} Q \Delta_{\zeta_{2}}\right)
\end{aligned}
$$

By considering Lemma (1), inequality (23) yields:

$$
\begin{aligned}
& \dot{V}(e(t))<\sum_{i=1}^{p_{1}} \mu_{i}\left(\zeta_{1}\right)\left(e_{\zeta_{1}}^{T}\left(\Gamma_{i}+P \bar{D}_{i} \mathscr{G}_{1} \bar{D}_{i}^{T} P+P \mathscr{F}_{1} P\right) e_{\zeta_{1}}+e_{\zeta_{2}}^{T} \mathscr{G}_{1}^{-1} e_{\zeta_{2}}+\right. \\
& \left.\Delta_{\zeta_{1}}^{T} \mathscr{F}_{1}^{-1} \Delta_{\zeta_{1}}\right)+\sum_{j=1}^{p_{2}} \vartheta_{j}\left(\zeta_{1}\right)\left(e_{\zeta_{2}}^{T}\left(\breve{\Gamma}_{j}+Q \breve{D}_{j} \mathscr{G}_{2} \breve{D}_{j}^{T} Q+Q \mathscr{F}_{2} Q\right) e_{\zeta_{2}}+e_{\zeta_{1}}^{T} \mathscr{G}_{2}^{-1} e_{\zeta_{1}}+\right. \\
& \left.\Delta_{\zeta_{2}}^{T} \mathscr{F}_{2}^{-1} \Delta_{\zeta_{2}}\right)
\end{aligned}
$$

where, $\mathscr{G}_{1}$ and $\mathscr{G}_{2}$ are positive definite matrices. Then, if inequality $\dot{V}(e(t))<0$ holds, one have

$$
\begin{aligned}
& \sum_{i=1}^{p_{1}} \mu_{i}\left(\zeta_{1}\right)\left(e_{\zeta_{1}}^{T}\left(\Gamma_{i}+P \bar{D}_{i} \mathscr{G}_{1} \bar{D}_{i}^{T} P+P \mathscr{F}_{1} P+\mathscr{G}_{2}^{-1}\right) e_{\zeta_{1}}+\Delta_{\zeta_{1}}^{T} \mathscr{F}_{1}^{-1} \Delta_{\zeta_{1}}\right)+ \\
& \sum_{j=1}^{p_{2}} \vartheta_{j}\left(\zeta_{1}\right)\left(e_{\zeta_{2}}^{T}\left(\breve{\Gamma}_{j}+Q \breve{D}_{j} \mathscr{G}_{2} \breve{D}_{j}^{T} Q+Q \mathscr{F}_{2} Q+\mathscr{G}_{1}^{-1}\right) e_{\zeta_{2}}+\Delta_{\zeta_{2}}^{T} \mathscr{F}_{2}^{-1} \Delta_{\zeta_{2}}\right)<0
\end{aligned}
$$

Let us define

$$
\left\{\begin{array}{c}
\Xi_{i}=\Gamma_{i}+P \bar{D}_{i} \mathscr{G}_{1} \bar{D}_{i}^{T} P+P \mathscr{F}_{1} P+\mathscr{G}_{2}^{-1} \\
\Xi_{j}=\breve{\Gamma}_{j}+Q \breve{D}_{j} \mathscr{G}_{2} \breve{D}_{j}^{T} Q+Q \mathscr{F}_{2} Q+\mathscr{G}_{1}^{-1}
\end{array}\right.
$$

thus

$$
\dot{V}(e(t))<0 \Leftrightarrow \begin{aligned}
& \dot{V}(e(t))<\sum_{i=1}^{p_{1}} \mu_{i}\left(\zeta_{1}\right)\left(e_{\zeta_{1}}^{T} \Xi_{i} e_{\zeta_{1}}+\Delta_{\zeta_{1}}^{T} \mathscr{F}_{1}^{-1} \Delta_{\zeta_{1}}\right)+ \\
& \sum_{j=1}^{p_{2}} \vartheta_{j}\left(\zeta_{1}\right)\left(e_{\zeta_{2}}^{T} \Xi_{j} e_{\zeta_{2}}+\Delta_{\zeta_{2}}^{T} \mathscr{F}_{2}^{-1} \Delta_{\zeta_{2}}\right)<0
\end{aligned}
$$

The inequality (26) is equivalent to:

$$
\begin{aligned}
& \dot{V}(e(t))<\sum_{i=1}^{p_{1}} \mu_{i}\left(\zeta_{1}\right)\left(e_{\zeta_{1}}^{T} \Xi_{i} e_{\zeta_{1}}+\Delta_{\zeta_{1}}^{T} \mathscr{F}_{1}^{-1} \Delta_{\zeta_{1}}\right) \\
& +\alpha\left(e_{\zeta_{1}} P e_{\zeta_{1}}+e_{\zeta_{2}} Q e_{\zeta_{2}}\right)-\alpha\left(e_{\zeta_{1}} P e_{\zeta_{1}}+e_{\zeta_{2}} Q e_{\zeta_{2}}\right)+ \\
& \sum_{j=1}^{p_{2}} \vartheta_{j}\left(\zeta_{1}\right)\left(e_{\zeta_{2}}^{T} \Xi_{j} e_{\zeta_{2}}+\Delta_{\zeta_{2}}^{T} \mathscr{F}_{2}^{-1} \Delta_{\zeta_{2}}\right)<0
\end{aligned}
$$

Then, one obtain:

$$
\begin{aligned}
& \dot{V}(e(t))<\sum_{i=1}^{p_{1}} \mu_{i}\left(\zeta_{1}\right)\left(e_{\zeta_{1}}^{T}\left(\Xi_{i}+\alpha P\right) e_{\zeta_{1}}+\Delta_{\zeta_{1}}^{T} \mathscr{F}_{1}^{-1} \Delta_{\zeta_{1}}\right) \\
& +\sum_{j=1}^{p_{2}} \vartheta_{j}\left(\zeta_{1}\right)\left(e_{\zeta_{2}}^{T}\left(\Xi_{j}+\alpha Q\right) e_{\zeta_{2}}+\Delta_{\zeta_{2}}^{T} \mathscr{F}_{2}^{-1} \Delta_{\zeta_{2}}\right) \\
& -\alpha\left(e_{\zeta_{1}} P e_{\zeta_{1}}+e_{\zeta_{2}} Q e_{\zeta_{2}}\right)<0
\end{aligned}
$$

Considering $e_{\zeta}(t)=\left[\begin{array}{ll}e_{\zeta_{1}}^{T}(t) & e_{\zeta_{2}}^{T}(t)\end{array}\right]^{T}$. The inequality (28) is equivalent to:

$$
\begin{aligned}
\dot{V}(t) \leq & \sum_{i=1}^{p_{1}} \mu_{i}\left(\zeta_{1}\right) e_{\zeta_{1}}^{T}\left(\Xi_{i}+\alpha P\right) e_{\zeta_{1}}+\sum_{j=1}^{p_{2}} \vartheta_{j}\left(\zeta_{1}\right)\left(e_{\zeta_{2}}^{T}\left(\Xi_{j}+\alpha Q\right) e_{\zeta_{2}}\right. \\
& -\alpha\left(e_{\zeta_{1}} P e_{\zeta_{1}}+e_{\zeta_{2}} Q e_{\zeta_{2}}\right)+\Delta_{\zeta_{1}}^{T} \mathscr{F}_{1}^{-1} \Delta_{\zeta_{1}}+\Delta_{\zeta_{2}}^{T} \mathscr{F}_{2}^{-1} \Delta_{\zeta_{2}}
\end{aligned}
$$

Considering

$$
\Psi=\left[\begin{array}{cc}
\sum_{i=1}^{p_{1}} \mu_{i}\left(\zeta_{1}\right) \Xi_{i}+\alpha P & 0 \\
0 & \sum_{j=1}^{p_{2}} \vartheta_{j}\left(\zeta_{1}\right) \Xi_{j}+\alpha Q
\end{array}\right]<0
$$

Then, the time derivative of the Lyapunov function (29) is then bounded as follows

$$
\dot{V}(e(t))<e_{\zeta}^{T} \Psi e_{\zeta}-\alpha e_{\zeta}^{T}(t) \mathscr{Q} e_{\zeta}(t)+\Delta_{\zeta_{1}}^{T} \mathscr{F}_{1}^{-1} \Delta_{\zeta_{1}}+\Delta_{\zeta_{2}}^{T} \mathscr{F}_{2}^{-1} \Delta_{\zeta_{2}}<0
$$

where, $\mathscr{Q}=\operatorname{diag}(P, Q)$. Now, if $e_{\zeta}^{T} \Psi e_{\zeta}<0$ then the inequality (30) can be bounded as follows

$$
\begin{aligned}
& \dot{V}(t) \leq-\alpha e_{\zeta}^{T}(t) \mathscr{Q} e_{\zeta}(t)+\Delta_{\zeta_{1}}^{T} \mathscr{F}_{1}^{-1} \Delta_{\zeta_{1}}+\Delta_{\zeta_{2}}^{T} \mathscr{F}_{2}^{-1} \Delta_{\zeta_{2}} \\
\leq & -\alpha\left(e_{\zeta_{1}}^{T} P e_{\zeta_{1}}+e_{\zeta_{2}}^{T} Q e_{\zeta_{2}}\right)+\Delta_{\zeta_{1}}^{T} \mathscr{F}_{1}^{-1} \Delta_{\zeta_{1}}+\Delta_{\zeta_{2}}^{T} \mathscr{F}_{2}^{-1} \Delta_{\zeta_{2}}
\end{aligned}
$$

which is equivalent to

$$
\dot{V}(t) \leq-\alpha V(t)+\Delta_{\zeta_{1}}^{T} \mathscr{F}_{1}^{-1} \Delta_{\zeta_{1}}+\Delta_{\zeta_{2}}^{T} \mathscr{F}_{2}^{-1} \Delta_{\zeta_{2}}
$$

It follows

$$
\begin{aligned}
& V(t) \leq V(0) e^{-\alpha t}+\mathscr{F}_{1} \int_{0}^{t} e^{-\alpha(t-s)}\left\|\Delta_{\zeta_{1}}(s)\right\|_{2}^{2} d s+\mathscr{F}_{2} \int_{0}^{t} e^{-\alpha(t-s)}\left\|\Delta_{\zeta_{2}}(s)\right\|_{2}^{2} d s \\
& \leq V(0) e^{-\alpha t}+\frac{\mathscr{F}_{1}}{\alpha}\left\|\Delta_{\zeta_{1}}(s)\right\|_{\infty}^{2}+\frac{\mathscr{F}_{2}}{\alpha}\left\|\Delta_{\zeta_{2}}(s)\right\|_{\infty}^{2}
\end{aligned}
$$

one obtains the inequality

$$
\left\|e_{\zeta}(t)\right\|_{2}^{2} \leq \frac{\lambda_{\max }(\mathscr{Q})}{\lambda_{\min }(Q)}\left(e^{-\alpha t}+\frac{\mathscr{F}_{1}}{\alpha}\left\|\Delta_{\zeta_{1}}(s)\right\|_{\infty}^{2}+\frac{\mathscr{F}_{2}}{\alpha}\left\|\Delta_{\zeta_{2}}(s)\right\|_{\infty}^{2}\right)
$$

By using the square root on (33), one obtains

$$
\left\|e_{\zeta}(t)\right\|_{2}^{2} \leq \sqrt{\frac{\lambda_{\max }(\mathscr{Q})}{\lambda_{\min }(\mathscr{Q})}}\left(e^{-\frac{\alpha}{2} t}+\sqrt{\frac{\mathscr{F}_{1}}{\alpha}}\left\|\Delta_{\zeta_{1}}(s)\right\|_{\infty}+\sqrt{\frac{\mathscr{F}_{2}}{\alpha}}\left\|\Delta_{\zeta_{2}}(s)\right\|_{\infty}\right)
$$

According to Lyapunov formulation of Input To State Practical Stability (ISpS), the states converge to a region which will be minimized in order to achieve a more accurate estimation of the states of the motorcycle longitudinal and lateral motions. This ball is smaller as the attenuation level of the transfer from $\Delta_{\zeta_{1}}(t), \Delta_{\zeta_{2}}(t)$ to the state estimation errors are smaller. To enhance the performances of the observer, a minimal values of these quantities are obtained by the following reasoning: Let us consider the quantity $\sqrt{\frac{\lambda_{\max }(\mathscr{Q})}{\lambda_{\min }(\mathscr{Q}) \alpha}} \leq \sqrt{\eta}$ where $\eta=$ $\operatorname{diag}\left(\eta_{1}, \eta_{2}\right), \eta_{i}$ is a positive scalar. It is then sufficient to minimize the term $\eta$ and assuming $\lambda_{\min }(\mathscr{Q}) \geq 1(\mathscr{Q}>I)$, one obtains:

$$
\sqrt{\frac{\lambda_{\max }(P)}{\alpha}} \leq \sqrt{\eta_{1}}, \quad \sqrt{\frac{\lambda_{\max }(Q)}{\alpha}} \leq \sqrt{\eta_{2}}
$$

which is transformed easily into:

$$
\left(\alpha \eta_{1}\right)^{2} I-P^{T} P>0, \quad\left(\alpha \eta_{2}\right)^{2} I-Q^{T} Q>0
$$

Using Shur's complement lemma:

$$
\left(\begin{array}{cc}
\alpha \eta_{1} I & P \\
P & \alpha \eta_{1} I
\end{array}\right)>0, \quad\left(\begin{array}{cc}
\alpha \eta_{2} I & Q \\
Q & \alpha \eta_{2} I
\end{array}\right)>0 P \geq I \quad Q \geq I
$$

Always in the purpose of minimizing the two quantities, in theorem 1 , the chosen objective function is a linear combination between $\eta_{1}$ and $\eta_{2}$. Now, using the convex sum propriety and the condition $\dot{V}(e(t))<0$ ( $\Psi<0$ holds). The condition $\Psi<0$ leads to the following optimization problem:

$$
\left[\begin{array}{cc}
\Xi_{1}+\alpha P & 0 \\
0 & \Xi_{2}+\alpha Q
\end{array}\right]<0
$$

with

$$
\begin{cases}\Xi_{1} & =\Gamma_{i}+P \bar{D}_{i} \mathscr{G}_{1} \bar{D}_{i}^{T} P+P \mathscr{F}_{1} P+\mathscr{G}_{2}^{-1} \\ \Xi_{2} & =\breve{\Gamma}_{j}+Q \breve{D}_{j} \mathscr{G}_{2} \bar{D}_{j}^{T} Q+Q \mathscr{F}_{2} Q+\mathscr{G}_{1}^{-1} \\ \Gamma_{i} & =\bar{\Phi}_{i}^{T} P+P \bar{\Phi}_{i}, \Gamma_{j}=\breve{\Phi}_{j}^{T} Q+Q+Q \breve{\Phi}_{j} \\ \bar{\Phi}_{i} & =\left(\bar{A}_{i}-\bar{L}_{i} \bar{C}\right), \breve{\Phi}_{j}=\left(\breve{A}_{j}-\breve{L}_{j} \breve{C}^{\prime}\right)\end{cases}
$$

Then, one have:

$$
\begin{aligned}
{\left[\Gamma_{i}+P \bar{D}_{i} \mathscr{G}_{1} \bar{D}_{i}^{T} P+P \mathscr{F}_{1} P+\mathscr{G}_{2}^{-1}+\alpha P\right] } & <0 \\
{\left[\breve{\Gamma}_{j}+Q \breve{D}_{j} \mathscr{G}_{2} \breve{D}_{j}^{T} Q+Q \mathscr{F}_{2} Q+\mathscr{G}_{1}^{-1}+\alpha Q\right] } & <0
\end{aligned}
$$

The two matrix inequalities are connected by $\mathscr{G}_{1}$ and $\mathscr{G}_{2}$. Using Schur Lemma (2), inequalities (39 yield to:

$$
\begin{gathered}
{\left[\begin{array}{cc}
\Gamma_{i}+\mathscr{G}_{2}^{-1}+\alpha P & P \bar{D}_{i}+P \mathscr{F}_{1} \\
\bar{D}_{i}^{T} P+\mathscr{F}_{1}^{T} P & -\mathscr{G}_{1}^{-1}
\end{array}\right]<0, \quad i=1, \ldots, p_{1}} \\
{\left[\begin{array}{cc}
\Gamma_{j}+\mathscr{G}_{1}^{-1}+\alpha Q & Q \breve{D}_{j}+Q \mathscr{F}_{2} \\
\breve{D}_{j}^{T} Q+\mathscr{F}_{2}^{T} Q & -\mathscr{G}_{2}^{-1}
\end{array}\right]<0, \quad j=1, \ldots, p_{2}}
\end{gathered}
$$

By using the definitions of the matrices $\Gamma_{i}$ and $\breve{\Gamma}_{j}$ and change of variables $K_{i}=P L_{i}, \breve{K}_{j}=Q \breve{L}_{j}$ and $\Omega_{1}=\mathscr{G}_{1}^{-1}$ 
and $\Omega_{2}=\mathscr{G}_{2}^{-1}$, where $\Omega_{1}$ and $\Omega_{2}$ are diagonal and positive definite matrices $R_{1}=P \mathscr{F}_{1}$ and $R_{2}=Q \mathscr{F}_{2}$. Finally, the gains of the interconnected observer are computed from the LMI conditions given in the following theorem.

Theorem 1: The state estimation error between the system and the interconnected observers converges asymptotically to zero if there exists two symmetric and definite matrices $P$ and $Q$, two diagonal positive matrices $\Omega_{1}$ and $\Omega_{2}$, given a positive scalars $\sigma$ and $\alpha$ and $a \in[0,1]$ and gains $K_{i}, \quad i=1, \ldots, p_{1}$ and $\breve{K}_{j}, \quad j=1, \ldots, p_{2}$ such that the LMI conditions:

$$
\min _{P, Q, \eta_{1}, \eta_{2}} a \eta_{1}+(1-a) \eta_{2}
$$

s.t.

$$
\begin{gathered}
{\left[\begin{array}{cc}
\bar{A}_{i}^{T} P+P \bar{A}_{i}-K_{i} \bar{C}-\bar{C}^{T} K_{i}^{T}+\Omega_{2}+\alpha P & P \bar{D}_{i}+R_{1} \\
\bar{D}_{i}^{T} P+R_{1}^{T} & -\Omega_{1}
\end{array}\right]<0} \\
{\left[\begin{array}{cc}
\breve{A}_{i}^{T} P+P \breve{A}_{i}-\breve{K}_{i} \breve{C}-\breve{C}^{T} \breve{K}_{i}^{T}+\Omega_{1}+\alpha Q & Q \breve{D}_{j}+R_{2} \\
\breve{D}_{j}^{T} Q+R_{2}^{T} & -\Omega_{2}
\end{array}\right]<0}
\end{gathered}
$$

The gains of the observer are obtained from the equations $L_{i}=P^{-1} K_{i}, \quad i=1, \ldots, p_{1}$ and $\breve{L}_{i}=Q^{-1} \breve{K}_{i}, \quad i=1, \ldots, p_{1}$.

\section{Observer Evaluation and Simulation}

The proposed observer is evaluated by co-simulation with Bikesim $^{\odot}$ software [3]. A PTWv model is chosen from the dataset Big Sport Baseline 8 bodies and default parameters. The simulations are carried out in two maneuver:

Test 1: Accelerate and brake in Alt 3 Road from FHWA (Federal Highway Administration) with a high friction coefficient $\mu=0.9$.

Test 2: An oncoming traffic with variable speed on handling road course.

The motorcycle system, requires three inputs: the rider's steering torque applied on the handlebars and the two braking torques applied on both front and rear wheels. In the following, if the actual state $v_{y}, v_{x}$ and $F_{y f}, F_{y r}$ and $F_{x f}, F_{x r}$ are unknown, the state estimation can be validated from lateral and longitudinal accelerations as follow:

$$
\begin{aligned}
& E q_{1}: \hat{a}_{y}=\frac{\left(\hat{F}_{y f}+\hat{F}_{y r}\right)}{M}, E q_{2}: \hat{a}_{y}=\hat{v_{y}}+v_{x} \hat{\dot{\psi}} \\
& E q_{3}: \hat{a}_{x}=\frac{\left(\hat{F}_{x f}+\hat{F}_{x r}\right)}{M}, E q_{4}: \hat{a}_{x}=\hat{\hat{v}_{x}}-v_{y} \hat{\dot{\psi}}+C_{d} / M \cdot \hat{v}_{x}^{2}
\end{aligned}
$$

\section{A. Accelerate and brake in longitudinal and lateral motion}

The simulations are carried out from FHWA, this maneuver demonstrates an acceleration and braking test includes three phases. The acceleration phase $(0 \prec t \prec 10(s))$, where only the drive torque $T$ is applied on the rear wheel. In the phase $(10 \prec t \prec 20(s)$ ), the main body is subject to lateral motion, imposed by the applied $\tau$. The braking phase $(20 \prec t \prec 30(s)$ ), where braking torque $\left(B_{f}, B_{r}\right)$ are applied to reduce the forward speed from $38 \mathrm{~m} / \mathrm{s}$ to $15 \mathrm{~m} / \mathrm{s}$. The inputs of the lateral and longitudinal models are the braking torques, drive engine and steering torque depicted in figure $3 \mathrm{a}$. The measured state used in the observer design are given in figures $3 \mathrm{~b}$. Also, the lateral and forward acceleration $\left(a_{x}\right.$ and $\left.a_{y}\right)$ are used in the observer design and used to validate the estimation of the unmeasured states. Estimation results of this scenario are

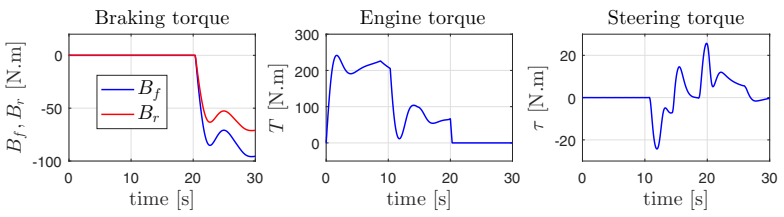

(a) Inputs $\left(B_{f}, B_{r}, T, \tau\right)$.
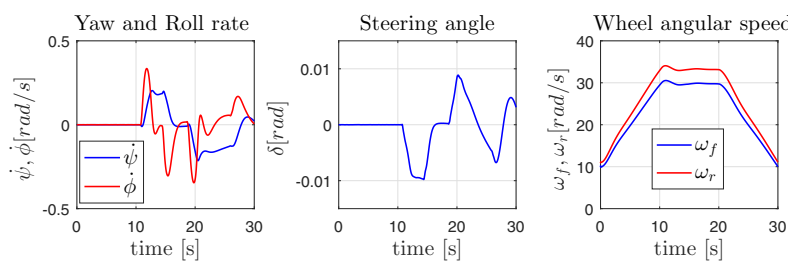

(b) BikeSim measured states $(\dot{\psi}, \dot{\phi}, \dot{\psi}, \delta)$

Fig. 3: Test 1: FHWA.
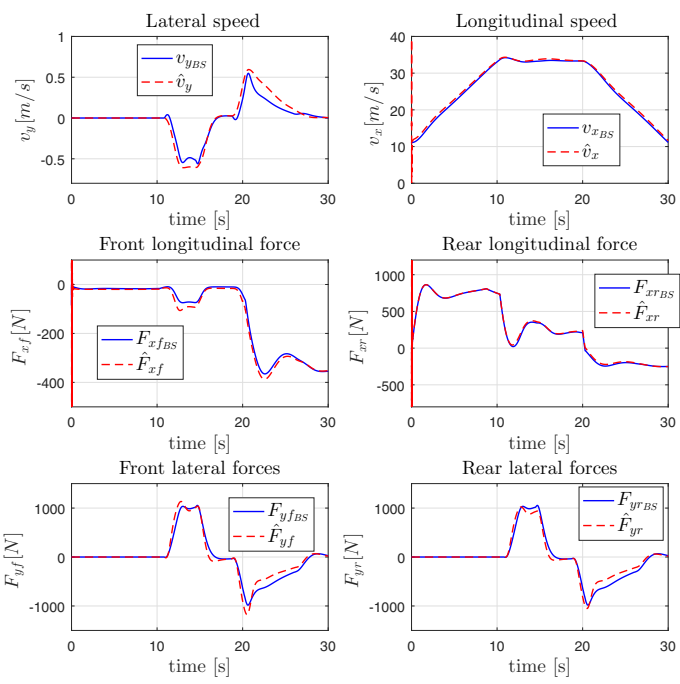

(a) Actual states (in blue) compared to estimated states (dashed red).
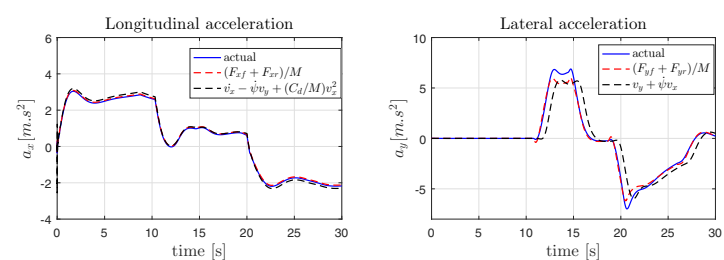

(b) Validation of the estimated states.

Fig. 4: Test 1: FHWA.

depicted on figure $4 \mathrm{a}$, which are the lateral and longitudinal speeds, the front and rear longitudinal forces, the front and rear cornering forces. From equation (44), the unmeasured state ( $v_{x}$, $v_{y}, F_{x_{i}}, F_{y_{i}}$ ) are validated and depicted in figure $4 \mathrm{~b}$.

\section{B. An oncoming traffic in road course}

In this test, the motorbike undergoes an oncoming traffic in road course with variable speed, on a handling flat road. The figure 5a shows the input signals in the longitudinal and 
lateral models whereas figures $5 \mathrm{~b}$ depict the measured states along the track. The unmeasured state are estimated, depicted in Fig. 6a, and validated in figure 6b. It can be appreciated

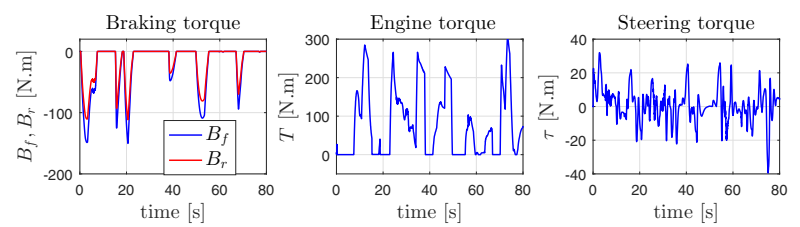

(a) Inputs $\left(B_{f}, B_{r}, T, \tau\right)$.
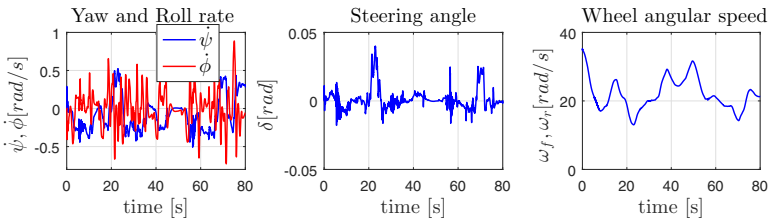

(b) Measured states.

Fig. 5: Test 2: Road course.
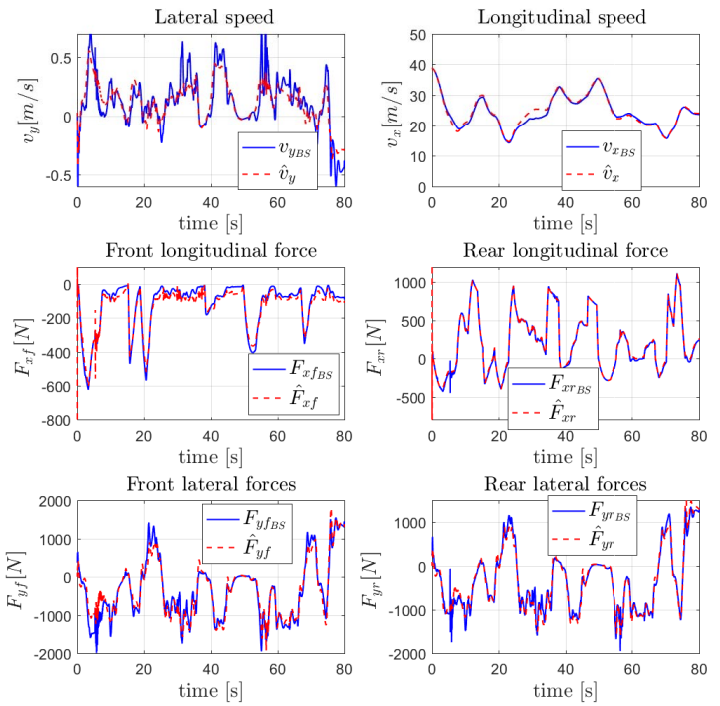

(a) Actual states (in blue) compared to estimated states (dashed red).
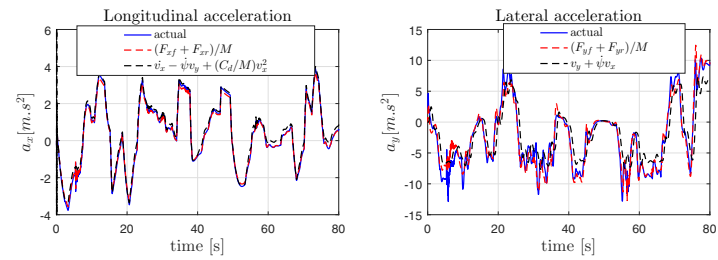

(b) Validation of the estimated states.

Fig. 6: Test 2: Road course.

that the proposed observer shows a good estimation accuracy and proves the reliability of the approach to estimate simultaneously the longitudinal and lateral dynamics states of the interconnected model. Figures illustrate that the observer rapidly and accurately estimates the state of the model with minimal error.

\section{CONCLUSION}

This paper deals with an interconnected observer formulation of motorcycle estimation problem is presented and evaluated throughout co-simulation with a high-end motorcycle simulation. First of all, the two sub-models of longitudinal and lateral motorcycle motion are transformed to interconnected TS sub-systems using polytopic approach. After, the observer has been designed subject to Lyapunov theory and the Input to State Practical Stability (ISpS) to guaranty boundness of the state estimation errors. Afterward, sufficient conditions for the existence of estimator are given in terms of LMIs. The observer allows the reconstruction of relevant non-measurable states of the PTWv: the forward speed and the longitudinal tire forces from the first sub-observer and lateral speed, roll angle and the cornering forces from the second sub-observer.

\section{REFERENCES}

[1] F. I. N. O. of Road Safety, "characteristics and causes of road accidents," Tech. Rep., 2010.

[2] B. Amans and M. Moutreuil, "Rider project . research on accidents involving powered two-wheelers final report n rider200503-10," French National Agency of Reaserch, Tech. Rep., 2005.

[3] R. Sharp, S. Evangelou, and D. J. Limebeer, "Advances in the modelling of motorcycle dynamics," Multibody system dynamics, vol. 12, no. 3, pp. 251-283, 2004.

[4] V. Cossalter, Motorcycle dynamics. Lulu. com, 2006.

[5] I. Boniolo, S. M. Savaresi, and M. Tanelli, "Roll angle estimation in two-wheeled vehicles," Control Theory Applications, IET, vol. 3, no. 1, pp. 20-32, 2009.

[6] A. Teerhuis and S. Jansen, "Motorcycle state estimation for lateral dynamics," Vehicle system dynamics, vol. 50, no. 8, pp. 1261-1276, 2012.

[7] I. Boniolo, S. M. Savaresi, and M. Tanelli, "Lean angle estimation in two-wheeled vehicles with a reduced sensor configuration," in Circuits and Systems (ISCAS), 2012 IEEE International Symposium on, pp. 2573-2576. IEEE, 2012.

[8] M. E.-H. Dabladji, D. Ichalal, H. Arioui, and S. Mammar, "Unknowninput observer design for motorcycle lateral dynamics: Ts approach," Control Engineering Practice, vol. 54, pp. 12-26, 2016.

[9] S. Evangelou, "Control and stability analysis of two-wheeled road vehicles," Ph.D. dissertation, University of London London, 2004.

[10] M. Corno, S. M. Savaresi, M. Tanelli, and L. Fabbri, "On optimal motorcycle braking," Control Engineering Practice, vol. 16, pp. 644 $-657,2008$.

[11] M. Corno, G. Panzani, and S. M. Savaresi, "Traction-control-oriented state estimation for motorcycles," IEEE Transactions on Control Systems Technology, vol. 21, no. 6, pp. 2400-2407, 2013.

[12] M. E.-H. Dabladji, D. Ichalal, H. Arioui, and S. Mammar, "On the estimation of longitudinal dynamics of powered two-wheeled vehicles," in Control Conference (ECC), 2015 European, pp. 921-926. IEEE, 2015.

[13] M. E.-H. Dabladji, D. Ichalal, H. Arioui, and S. Mammar, "On the algebraic estimation of whole two-wheeled vehicles dynamics via high order sliding mode differentiators," in International Conference on Automatic control, Telecommunication and Signals, 2015.

[14] R. S. Sharp, "The stability and control of motorcycles," Journal of mechanical engineering science, vol. 13, no. 5, pp. 316-329, 1971.

[15] K. Tanaka and H. O. Wang, Fuzzy control systems design and analysis: a linear matrix inequality approach. John Wiley \& Sons, 2004.

[16] S. Boyd, L. El Ghaoui, E. Feron, and V. Balakrishnan, Linear Matrix Inequalities in System and Control Theory, P. SIAM, Ed. SIAM ed., 1994.

[17] M. Lazar, D. Munoz de la Pena, W. Heemels, and T. Alamo, "On input-to-state stability of min-max nonlinear model predictive control," Systems \& Control Letters, vol. 57, 2008. 